\title{
LIN-41/TRIM71: emancipation of a miRNA target
}

\author{
Matyas Ecsedi ${ }^{1,2}$ and Helge Großhans ${ }^{1,3}$ \\ ${ }^{1}$ Friedrich Miescher Institute for Biomedical Research, CH-4058 Basel, Switzerland; ${ }^{2}$ University of Basel, CH-4003 Basel, \\ Switzerland
}

lin-41 (lineage variant 41)/TRIM71 (tripartite motif 71) is well known for being a conserved target of the let-7 (lethal 7) microRNA (miRNA), a regulatory relationship found in animals evolutionarily as distant as Caenorhabditis elegans and humans. It has thus been studied extensively as a model for miRNA-mediated gene silencing. In contrast, the developmental and molecular functions of LIN41 have historically received less attention. However, LIN41 proteins are now emerging as important regulators of cell proliferation and differentiation in stem and progenitor cells. Moreover, LIN41's functions appear to involve two distinct molecular activities; namely, protein ubiquitylation and post-transcriptional silencing of mRNAs. Thus, LIN41 is ready for a scientific life of its own.

Caenorhabditis elegans lin-41 (lineage variant 41) was originally discovered more than a decade ago as a target of the highly conserved let-7 (lethal 7) microRNA (miRNA) (Reinhart et al. 2000; Slack et al. 2000). This was also the time when miRNAs were beginning to emerge as a large and important class of regulators of gene expression in plants and animals (Lagos-Quintana et al. 2001; Lau et al. 2001; Lee and Ambros 2001; Reinhart et al. 2002). However, very few miRNA targets had been validated, and among the known targets, lin-41 stood out in that orthologous proteins could be identified in other organisms, including mice and humans (Slack et al. 2000). Yet more strikingly, even the regulation by let-7 was conserved for these orthologs (Kloosterman et al. 2004; Schulman et al. 2005; Lin et al. 2007). It is thus hardly surprising that lin-41 quickly became an intensely studied model miRNA target. Somewhat paradoxically, then, a focus on the mechanism of lin-41 regulation by let-7 also meant that our understanding of the molecular and developmental functions of LIN-41 itself has lagged behind. Recent studies have begun to change this and identified LIN-41 and the orthologous tripartite motif 71 (TRIM71) proteins as regulators of stem and progenitor

[Keywords: RING domain; development; differentiation; stem cell; selfrenewal; ubiquitin]

${ }^{3}$ Corresponding author

E-mail helge.grosshans@fmi.ch

Article is online at http://www.genesdev.org/cgi/doi/10.1101/gad.207266.112. cell proliferation and differentiation that can silence mRNA and drive protein ubiquitylation (Rybak et al. 2009; Chang et al. 2012; J Chen et al. 2012; Loedige et al. 2012). Here we discuss these exciting novel insights into the molecular and developmental biology of lin-41. For simplicity, we avoid, in the following, species-specific nomenclatures and refer to the gene and mRNA as LIN41 and the protein product as LIN41, respectively.

\section{Prelude: LIN41 as a target of the let-7 miRNA}

LIN41 is a member of the TRIM-NHL family of proteins (for review, see Wulczyn et al. 2011). The family name derives from the tripartite motif of RING /really interesting new gene) finger, B-box(es), and coiled-coil domain (accordingly also named RBCC) and, typically, six NHL repeats at the $\mathrm{C}$ terminus (Fig. 1). (Here, NHL stands for NCL-1, HT2A2, and LIN-41, after the proteins in which this motif was first discovered [Slack and Ruvkun 1998].) Initially, LIN41 was identified as a suppressor of phenotypes caused by a let-7 loss-of-function (lf) mutation as well as a regulator of temporal cell fates in C. elegans larvae (Slack et al. 2000), as discussed in more detail in a later section. The genetic data supported let-7 functioning as a negative regulator of LIN41, and parallel work identified let-7 as a short noncoding RNA (Reinhart et al. 2000), now known as a miRNA, which had potential to bind to partially complementary sites in the LIN41 3' untranslated region (UTR). Subsequent studies could confirm direct regulation of LIN41 by let-7 and identified sequence elements that generate functional let-7 target sites (Vella et al. 2004a, b; Long et al. 2007) and revealed LIN41 mRNA degradation (Bagga et al. 2005) and translational repression (Ding and Großans 2009) as modes of let-7 activity. Collectively, these findings were highly influential in building a general framework for our understanding of miRNA function, helped in part by the fact that let-7 sequence and function appeared highly conserved in animals. Specifically, let-7 orthologs, known as let-7a in some organisms, are $100 \%$ conserved in sequence and present in most animals (Pasquinelli et al. 2000; Lagos-Quintana et al. 2002). Moreover, let-7 was shown to repress fly (O'Farrell et al. 2008), zebrafish (Kloosterman et al. 2004), mouse (Kanamoto et al. 2006; Rybak et al. 2009), chicken (Kanamoto et al. 2006), and 


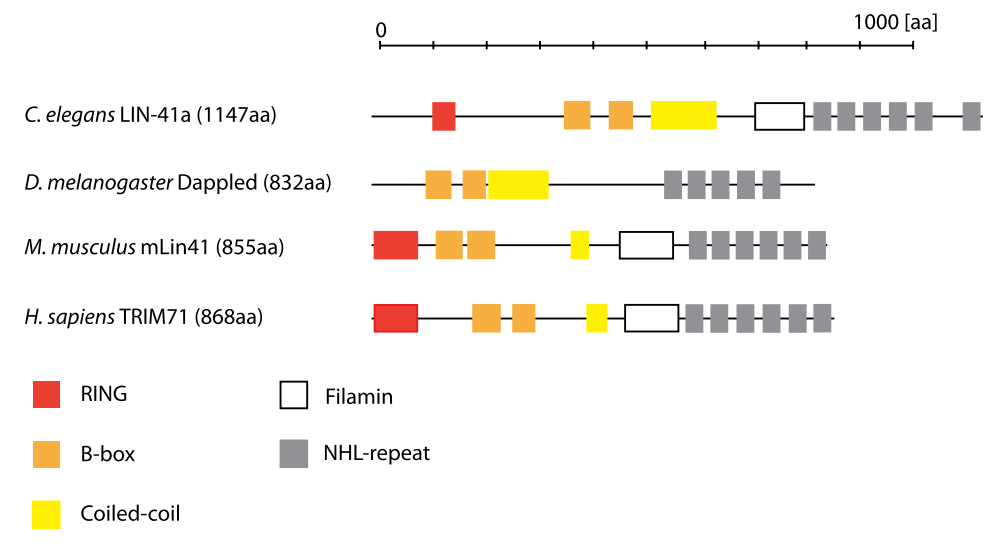

Figure 1. Domain architecture of LIN41 proteins from different species. The indicated domains were identified using SMART (http://smart.embl-heidelberg.de) and CDART (http://www.ncbi.nlm.nih.gov/Structure/ lexington/lexington.cgi) algorithms. Domain sizes are approximately to scale. Note that two isoforms have been reported for C. elegans, LIN41a and LIN41B, which only differ by three amino acids (Slack et al. 2000). human (Lin et al. 2007) LIN41 orthologs in 3' UTR reporter assays. Finally, ectopic expression of let-7 decreased endogenous LIN41 protein levels in a mouse embryonic carcinoma cell line (Rybak et al. 2009).

Surprisingly then, whereas extensive research on let-7 over the past decade has established it as a regulator of a wide range of processes in development (Mondol and Pasquinelli 2012), metabolism (Zhu et al. 2011), and human disease (Büssing et al. 2008), a contribution of LIN41 repression to these let-7 functions has not been investigated. This is despite the fact that the evolutionary conservation of LIN41 regulation by let-7 makes a strong case for LIN41 being a key let-7 effector, even when taking into account that most experiments involved the use of reporters and/or let-7 overexpression rather than regulation of endogenous LIN41 under physiological conditions. By extension, the evident importance of LIN41 regulation suggests that LIN41's yet elusive molecular functions might have significant impact on cellular homeostasis, and this idea is strongly supported by recently published data discussed in the following sections.

\section{An emerging theme: LIN41 controls cell proliferation and differentiation in vertebrate and invertebrate development}

C. elegans seam cells divide in an asymmetric, stem celllike fashion in a characteristic pattern during each larval stage (Sulston and Horvitz 1977). At the larval-to-adult (L/A) transition, the seam cells exit the cell cycle, fuse, and differentiate to secrete specialized collagenous structures called alae. The timing of these events is governed by the heterochronic pathway (Ambros and Horvitz 1984). Reduced levels of LIN41 lead to precocious execution of the L/A transition, as evidenced, for instance, by alae secretion and cell cycle exit in larvae. In contrast, LIN41 overexpression-or let-7lf-results in a retarded L/A transition, which promotes continued division of even adult seam cells, while preventing them from fusing and secreting alae (Reinhart et al. 2000; Slack et al. 2000). Epistasis analysis placed LIN41 downstream from the let7 miRNA and upstream of the lin-29 zinc finger transcription factor (Slack et al. 2000). Indeed, it has been suggested that let-7 acts primarily through LIN41 because RNAi-mediated depletion of LIN41, but not another putative target, $h b l-1$, almost completely suppressed aberrant seam cell divisions in let-7(0) mutant adult animals (Vadla et al. 2012). However, we note that lin-41lf; let-7(0) double-mutant animals generate alae, like wild-type animals, only at the L4-to-adult molt (Slack et al. 2000). In contrast, precocious alae formation at the L3 molt, a hallmark of lin-41lf single-mutant animals, is rare (Slack et al. 2000). These observations are inconsistent with the notion of a simple, linear pathway. It is therefore possible that distinct let-7 targets are key to regulating proliferation and differentiation, respectively, although this remains to be determined.

Although the hypodermis is the tissue where LIN41 function has been studied in most detail, LIN41 appears to play broader roles in C. elegans development. In addition to the hypodermis, a GFP-LIN41 fusion protein was observed in neurons, muscle cells, and the somatic gonad, in all of which it might accumulate in a let-7insensitive manner (Slack et al. 2000). As LIN41 mutant worms display gut defects (Del Rio-Albrechtsen et al. 2006) and are sterile due to a failure to produce oocytes (Slack et al. 2000), widespread expression appears functional, although the basis of the respective phenotypes remain to be determined. In C. elegans males, LIN41 further functions in morphogenesis of the tail tip. This process is temporally regulated (Nguyen et al. 1999) and highly sensitive to LIN41 dosage, as demonstrated by graded RNAi and allelic complementation studies (Del Rio-Albrechtsen et al. 2006). Similar to the hypodermis, reduced $L I N-41$ activity results in precocious, increased activity in retarded phenotypes (Del Rio-Albrechtsen et al. 2006). Mutations altering the LIN41 N terminus outside annotated domains cause the male tail tip but no hypodermal phenotypes, suggesting either a different threshold or, alternatively, different functions or interaction partners of LIN41 in these two tissues. Indeed, LIN29 , which we discuss in more detail in a later section, is a major effector of LIN41 functions in the hypodermis but dispensable in the tail (Del Rio-Albrechtsen et al. 2006).

The conservation of LIN41 sequence and regulation by let-7 in various animals as well as the fact that LIN41 expression is temporally and spatially regulated during development in animals where this has been investi- 
gated, strongly imply that LIN41 proteins have important functions in a wide range of animals and developmental contexts. This notion is supported by studies of specific loss-of-function phenotypes in zebrafish (Lin et al. 2007) and mice (Maller Schulman et al. 2008; J Chen et al. 2012). In zebrafish, knockdown of LIN41 leads to partially penetrant embryonic lethality, with surviving embryos showing developmental abnormalities such as a short trunk, abnormal yolk shape, and an S-shaped tail (Lin et al. 2007). Mice carrying a homozygous LIN41 "gene trap" mutation, which depletes LIN41 by providing a strong splice acceptor site that will cause endogenous LIN41 transcripts to missplice and terminate prematurely, start to die at around embryonic day 8.5 (E8.5), and all embryos are dead by E13.5-E16.5 (Maller Schulman et al. 2008; J Chen et al. 2012). The most striking phenotype of these LIN411f mice is a neural tube closure defect (Maller Schulman et al. 2008; J Chen et al. 2012), although this is apparently not the cause of lethality (Maller Schulman et al. 2008). J Chen et al. (2012) could subsequently pinpoint decreased proliferation rate and increased differentiation of neural progenitor cells, caused at least in part through defects in fibroblast growth factor (FGF) signaling, as the basis of the neural tube closure defect.

It is also in mice where LIN41 expression has been studied in the most detail, including by analysis of endogenous promoter activity (Maller Schulman et al. 2008; Yu et al. 2010; J Chen et al. 2012), mRNA in situ hybridization (Lancman et al. 2005; Schulman et al. 2005), immunohistochemistry (Rybak et al. 2009; Yu et al. 2010), and Western blot analysis (Rybak et al. 2009; Yu et al. 2010; J Chen et al. 2012). Generally, LIN41 expression decreases over development (Schulman et al. 2005; Yu et al. 2010; J Chen et al. 2012). In particular, following ubiquitous expression early, LIN41 transcription becomes gradually restricted to some neural tissues and the limb buds during embryogenesis (Maller Schulman et al. 2008). In neural tissue, LIN41 protein levels, measured by Western blotting, are high in neuroepithelial cells of early embryos (until E11.5) but undetectable as neurogenesis-and thus neural differentiation-proceeds (J Chen et al. 2012).

LIN41 expression not only correlates temporally with proliferative processes, but is also generally high in the proliferative, progenitor compartments of tissues where this has been characterized in more detail. Whole-mount staining of early embryos at E7 showed that LIN41 distribution is similar to that of the pluripotency factor Oct4, with staining in the embryonic ectoderm and, less strongly, the ectoplacental cone (Rybak et al. 2009). LIN41 was also detected in the interfollicular stem cells of both embryonic and adult epidermis as well as in postnatal testis, where it was found in gonocytes, early spermatogonia, and spermatocytes (Rybak et al. 2009). Interestingly, LIN41 mRNA or protein and let-7 display largely inverse expression patterns in skin and testis, with let-7 staining, for instance, being visible in the subrabasal cell layer rather than the basal stem cell layer, where LIN41 accumulates (Rybak et al. 2009). Taken together with the demonstration that LIN41 levels in mouse embryonic stem (mES) cells decrease during differentiation in parallel with up-regulation of let-7 (Chang et al. 2012), this suggests that let-7 plays a major role in shaping LIN41 expression during mouse-and, possibly more global, animal-development.

At the same time, it is worth keeping in mind that even for a notorious miRNA target such as LIN41, regulation by a miRNA is just one among many regulatory inputs. For instance, whole-mount in situ hybridization against endogenous LIN41 mRNA in FGF signaling-deficient mice suggests that expression of LIN41 in limb buds is dependent on FGF signaling, similar to the situation in chickens in which Shh and Fgf signaling induces LIN41 expression (Lancman et al. 2005). Interestingly, LIN41 itself appears to promote FGF signaling during mouse embryonic brain development (J Chen et al. 2012), which implies that LIN41 might stimulate its own expression. Furthermore, the LIN41 promoter and first intron contain three putative E-boxes that can be bound by the MYC transcription factor in cell lines (YL Chen et al. 2012). In luciferase reporter experiments, these elements can mediate transactivation of the LIN41 promoter by ectopic MYC expression (YL Chen et al. 2012). Although the physiological significance of this finding is not clear, MYC is, similar to LIN41, highly expressed and has an important function in ES cells (Kim et al. 2010). Moreover, in addition to transcriptionally activating LIN41, MYC relieves it from post-transcriptional repression by repressing let-7 directly (Chang et al. 2008) and through increased transcription of the let-7 inhibitor LIN28 (Chang et al. 2009; Dangi-Garimella et al. 2009). This exquisite regulation by at least two different mechanisms might point to a need to ensure sufficiently high LIN41 expression in ES cells in a robust and faithful manner.

Taken together, LIN41 expression in mice and worms appears to be a marker of a uni- or multipotent selfrenewing state. Given this expression pattern as well as the functions of LIN41 in mouse neural progenitor (J Chen et al. 2012) and C. elegans seam cells (Slack et al. 2000), an emerging principle of LIN41 activity in developmental processes and tissues seems to be promotion of proliferation and inhibition of differentiation. Consistent with this notion, LIN41 has been suggested to have a tumor-promoting role in human hepatocellular carcinoma (HCC): It is overexpressed in many tumor samples, and high levels correlate with poor patient survival (YL Chen et al. 2012). Moreover, LIN41 overexpression in HCC cell lines resulted in increased tumor growth in a subcutaneous xenograft model, which can probably be explained by an increased proliferation rate (YL Chen et al. 2012). In this regard, LIN41 may generally, in diverse organisms and developmental events, function as an important effector of the LIN28-let-7 "switch" between a self-renewing, proliferative stem cell or progenitor state, and differentiation (Melton et al. 2010). This idea is consistent with available data indicating that LIN28, a small RNA-binding protein, promotes proliferation and represses differentiation at least in part through repressing let-7 biogenesis (Rybak et al. 2008; Viswanathan et al. 
2008; Vadla et al. 2012) and that let-7 conversely promotes differentiation and represses proliferation while repressing LIN41. How LIN41 in turn controls the two processes of differentiation and proliferation is less clear, and we discuss in the next section potential molecular targets of, and mechanisms of regulation by, LIN41 (Fig. 2).

\section{A multitude of targets: cell cycle mRNAs and a signaling protein as LIN41 effectors}

Genetic analysis in C. elegans identified lin-29 as a factor functioning downstream from lin-41 in the control of differentiation and proliferation of seam cells (Slack et al. 2000). LIN-29 is a transcription factor and appears to provide a direct link to the cell cycle machinery, as it can stimulate transcription of the cell cycle inhibitor cki-1 (Hong et al. 1998). Moreover, LIN-29 promotes cell differentiation, although, with the exception of collagen genes (Rougvie and Ambros 1995), the relevant targets in
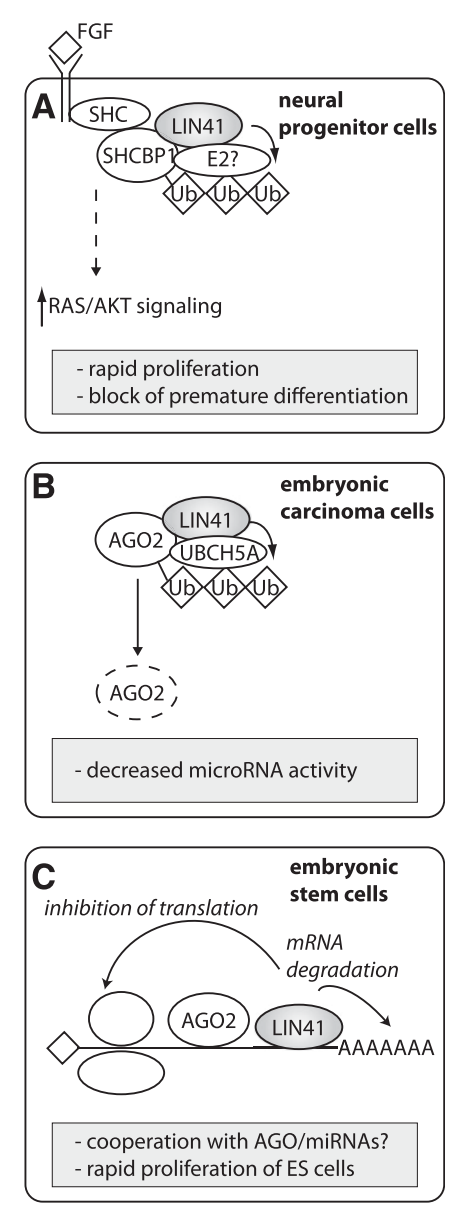

Figure 2. Proposed models of LIN41 function. (A) LIN41 ubiquitylates SHCBP1 to augment FGF signaling, increase proliferation rate, and inhibit premature differentiation in neural progenitor cells (J Chen et al. 2012). (B) LIN41 ubiquitylates AGO, inducing its degradation by the proteasome, leading to decreased miRNA activity (Rybak et al. 2009). (C) LIN41 induces degradation and translational inhibition of target mRNAs such as CDKN1a, promoting rapid proliferation of ES cells (Chang et al. 2012; Loedige et al. 2012). See the text for details. this process largely remain to be established. The fact that lin-29 mRNA accumulates two larval stages before LIN-29 protein becomes detectable (Rougvie and Ambros 1995; Bettinger et al. 1996), that LIN41lf mutations cause precocious LIN-29 accumulation in seam cells (Slack et al. 2000), and that a GFP-LIN41 fusion protein localizes to the cytoplasm (Slack et al. 2000) was taken to speculate that LIN41 could be a translational repressor of lin-29 (Slack et al. 2000).

Although experimental data for such an activity of LIN41 on lin-29-and, in fact, for lin-29 as a direct target of LIN41-are still lacking, evidence is now accumulating that LIN41 proteins are indeed post-transcriptional, potentially translational, silencers of mRNAs. Consistent with this notion, modulation of LIN41 levels changes the accumulation of numerous mRNAs and proteins in mouse and human cells, and in several instances, the $3^{\prime}$ UTRs of potential target genes are sufficient to recapitulate these effects for target reporters (Chang et al. 2012; Loedige et al. 2012). Moreover, LIN41 can directly repress translation of a luciferase reporter mRNA when artificially tethered to its 3' UTR of a luciferase reporter (Chang et al. 2012; Loedige et al. 2012). Silencing of either type of reporter is typically accompanied by some decrease in mRNA levels (Loedige et al. 2012). Finally, for a subset of these targets, it was shown that ectopically expressed LIN41 could coimmunoprecipitate the relevant endogenous mRNAs (Loedige et al. 2012). Thus, at least when expressed in HEK293 or mES cells, LIN41 displays several hallmarks of a translational repressor, although the mechanisms of repression remain to be elucidated.

How is LIN41 recruited to mRNAs? The LIN41 paralog and translational repressor BRAT might provide a model, as it is thought not to bind mRNAs directly, but rather in a complex with the pumilio protein PUF and the unspecific RNA-binding protein Nos (Sonoda and Wharton 2001). However, although both the $h b$ mRNA and the cyclin B 3' UTR contain Nos response elements (NREs), which recruit a NOS/PUF complex, BRAT only binds to the complex on the $h b$ NRE (Sonoda and Wharton 2001). Thus, mRNA features such as specific sequences or secondary structures seem to contribute to BRAT recruitment. It has not been possible so far to establish the requirements of target repression by LIN41. Although it interacts with several RNA-binding proteins, these interactions are mostly RNA-dependent (Loedige et al. 2012) and thus presumably not responsible for recruitment of LIN41 to RNA. Moreover, human pumilio proteins are not required for LIN41 activity in HEK293 cells, and on the mRNA side, there is no apparent consensus sequence or binding motif that would explain the interaction with LIN41 (Loedige et al. 2012).

Insights into LIN41's mode of action come from structure-function analyses. The coiled-coil and the filamin domain mediate mRNA repression in tethering assays but alone are not sufficient to recapitulate lin-41 activity in the context of a free mRNA (Loedige et al. 2012). Moreover, whereas a construct lacking the TRIM domain (i.e., RING, B-box, and coiled-coil domains) can coimmunoprecipitate LIN41 target mRNAs, a construct lacking 
the NHL domain cannot (Loedige et al. 2012). Combined with the fact that NHL domains are structurally similar to the more widely studied WD40 propellers, which have been shown to mediate both protein-protein interaction (Xu and Min 2011) and RNA binding (Lau et al. 2009; Castello et al. 2012), it seems possible that the NHL domain could mediate specific, and possibly direct, binding of target mRNAs. A domain swap experiment with Trim32 supports a key role of the NHL repeats in determining target specificity: Trim32 containing the NHL repeats of LIN41, but not wild-type Trim32, represses luciferase reporters containing the $3^{\prime}$ UTRs of LIN41 targets, whereas LIN41 containing Trim32 NHL repeats is inactive (Loedige et al. 2012).

Is mRNA silencing a major activity of LIN41 in regulating cell differentiation and proliferation in development? In support of this notion, the cyclin-dependent kinase inhibitor Cdkn1a was identified as a LIN41 target in mES cells, where LIN41 promotes G1-to-S-phase transition and thus increases proliferation (Chang et al. 2012). Specifically, Cdknla levels were increased when LIN41 was depleted by siRNAs, and combined knockdown of LIN41 and Cdkn1a rescued the slow proliferation rate as well as the G1-phase accumulation of LIN41-deficient cells (Chang et al. 2012). These data are consistent with, although not a direct proof of, LIN41 regulating cell proliferation in these cells through repression of Cdkn1a.

Loedige et al. (2012) sought to obtain a more global picture of the effects of LIN41 on gene expression and found that ectopic expression of LIN41 in HEK293 cells caused the expression levels of $>800$ genes to change significantly, whereas knockdown of LIN41 in mES cells affected $\sim 100$ genes. The $3^{\prime}$ UTRs of selected dysregulated genes were sufficient to confer post-transcriptional regulation by LIN41 to a reporter gene, with targets thus validated including genes with cell cycle-promoting but also inhibitory roles. For instance, the retinoblastomalike transcription factors Rbll and Rbl2 as well as E2F7 are inhibitors of the cell cycle; their repression might thus explain the increased proliferation rate observed in ES cells with high levels of LIN41. On the other hand, LIN41 also repressed positive regulators of the cell cycle such as cyclin D and cyclin E or Myb. The significance of these findings is currently not clear. It is also currently unknown which target mRNAs, if any, might mediate LIN41's effects on differentiation.

In addition to regulating mRNAs, mouse LIN41 was recently shown to bind to and stabilize Shcbp1 protein in mouse neural progenitor cells. Shcbpl is a putative mediator of FGF signaling, and Shcbp1 knockdown impairs FGF signaling in neural progenitor cells (J Chen et al. 2012). FGF signaling, like LIN41 activity, promotes neural precursor proliferation and represses differentiation (Guillemot and Zimmer 2011). Moreover, neuroepithelial cells from LIN41-deficient mice have decreased activity of the FGF pathway in vivo and are hyporesponsive to FGF stimulation in vitro (J Chen et al. 2012). Although it remains to be formally demonstrated that LIN41 affects FGF signaling by stabilizing SHCBP1 in neural progenitor or other cells, it therefore seems possible that a major func- tion of LIN41 in this developmental context is to promote FGF signaling to control cell proliferation and differentiation status.

Finally, as we discuss next, LIN41 has been shown to polyubiquitylate Argonaute (Ago), the core component of the miRNA-induced silencing complex (miRISC), which mediates mRNA degradation and translational repression by miRNAs. Since global impairment of miRNA activity has been shown in several examples to promote cell proliferation (Kumar et al. 2007; Hill et al. 2009; Melo et al. 2009, 2010), it is conceivable that ubiquitylationdependent Ago degradation would mediate (some of) LIN41's effects on cell proliferation and differentiation.

\section{LIN41 and miRNAs: a complex relationship}

Several TRIM-NHL family members have been found to regulate gene expression by modulating the miRNA pathway, suggesting a model of how LIN41 could regulate targets such as lin-29. For instance, C. elegans nhl-2 (Hammell et al. 2009), Drosophila mei-P26 (Neumüller et al. 2008), and mouse Trim32 (Schwamborn et al. 2009) influence miRNA activity either negatively or positively by interacting with Ago proteins. Intriguingly, a proteomic analysis identified LIN41 in immunoprecipitates of C. elegans Dicer, the enzyme that processes precursor miRNAs to mature miRNAs (Duchaine et al. 2006). However, this interaction has not been examined in great detail, and a functional relevance remains to be determined. In contrast, an interaction between LIN41 and Ago has been observed in various human and mouse cell lines. Specifically, several studies showed that LIN41 localizes at least in part to P-bodies, as assessed by immunofluorescence staining of endogenous LIN41 in KH2 ES cells (Chang et al. 2012) and embryonic carcinoma cells (Rybak et al. 2009) or staining tagged and overexpressed LIN41 in HEK293 (Loedige et al. 2012) and HeLa cells (Rybak et al. 2009). LIN41 not only resides in these sites of mRNA storage and degradation where Ago is also found (Kulkarni et al. 2010), but in fact physically interacts with Ago (Rybak et al. 2009; Chang et al. 2012; J Chen et al. 2012; Loedige et al. 2012), albeit in a largely RNA-dependent manner (Loedige et al. 2012).

Functionally, LIN41 was found to promote Ago ubiquitylation in vitro and in mouse embryonic carcinoma cells, where this resulted in proteasome-mediated Ago decay (Rybak et al. 2009). Repressive effects of LIN41 on Ago levels and on the function of several miRNAs have recently also been reported for HCC cell lines, although an involvement of Ago ubiquitylation was not examined in this study (YL Chen et al. 2012). Irrespective of mechanism, the observation of altered Ago levels raised the attractive hypothesis that LIN41, by antagonizing miRNA activity globally, could constitute a double-negative feedback loop with let-7 to create a bistable switch at the crossroad between the stem cell state and differentiation. The notion also provided an alternative explanation to earlier genetic data, which had shown that LIN41 depletion in worms could suppress developmental phenotypes seen with reduced activity of core components of 
the miRNA pathway (Grishok et al. 2001; Büssing et al. 2010). Since these phenotypes, vulval bursting and alae defects, are the same that are also found in let-7lf animals, it was originally assumed that the let-7 defect was predominant in the miRNA pathway mutant animals and accordingly suppressed upon LIN41 knockdown. The new data (Rybak et al. 2009) offered the alternative possibility that also in C. elegans, LIN41 could more directly modulate miRNA activity.

However, although ubiquitylation activity of LIN41 on Ago was confirmed in an independent study (Loedige et al. 2012), the functional consequences remain unclear. In particular, no changes in Ago2 levels or stability could be observed upon LIN41 knockdown in mES cells (Chang et al. 2012) or LIN41 overexpression in HEK293 cells (Loedige et al. 2012). Similarly, there was no difference in Ago2 levels between a wild-type and a LIN41-overexpressing neural progenitor cell line (J Chen et al. 2012). More importantly, neural progenitor cells of wild-type and LIN411f mice had similar Ago2 levels and ubiquitylation patterns in vivo (J Chen et al. 2012).

Although it is unclear why the results in the earlier (Rybak et al. 2009) and later (Chang et al. 2012; J Chen et al. 2012; Loedige et al. 2012) studies differ, collectively, these data indicate that regulation of Ago2 levels and thus global miRNA activity through polyubiquitylation by LIN41 does not seem to be a general phenomenon. As both studies that observed Ago2 decline upon LIN41 overexpression used transformed cell lines (Rybak et al. 2009; YL Chen et al. 2012), different LIN41 cofactors, altered baseline Ago2 stability, or levels in malignant cells might explain the observed differences.

Regardless of the possibility that LIN41 may alter miRNA activity globally, several LIN41 targets are also under miRNA control, and it has been suggested that similar to NHL-2, LIN41 and miRNAs may collaborate in target repression. For instance, in mES cells, Cdkn1a is regulated by both miR-302 and LIN41 via its 3' UTR. Consistent with LIN41 activity depending on miR-302 activity and vice versa, a reporter containing a 59-nucleotide (nt) fragment of the Cdkn1a 3' UTR that contained a validated miR-302-binding site was equally desilenced whether LIN41 and miR-302 were inactivated individually or jointly (Chang et al. 2012). Moreover, whereas LIN41 overexpression in wild-type ES cells promoted their proliferation, this effect was abrogated in $\operatorname{Dgcr} 8$ knockout cells, which lack most miRNAs (Chang et al. 2012). In contrast, examination of endogenous Cdkn1a mRNA showed that joint depletion of LIN41 and miR-302 additively stabilized its levels, which would argue against their cooperation (Chang et al. 2012). However, Cdknla protein levels were not examined in this experiment, whereas the reporter assay determined luciferase activity, which would integrate both mRNA and protein level changes. An intriguing but speculative possibility could be that LIN41 and miR-302 activities on mRNA levels are independent, whereas further repression of protein accumulation might require a cooperative mechanism.

In contrast to the findings on $C d k n 1 a$ regulation, examination of a larger set of LIN41 targets revealed that many of these remained unchanged upon Ago depletion (Loedige et al. 2012), suggesting that LIN41 can silence them independently of miRNA activity or can at least tolerate compromised miRNA activity. Moreover, ectopic expression of both miR-302 and LIN41 in HEK293 cells additively silenced an E2F7 reporter (Loedige et al. 2012). Although the latter experiment leaves open the possibility that LIN41 and miRNAs cooperate under physiological conditions with presumably lower endogenous expression levels, these results show that LIN41 does not, per se, require miRNAs to achieve mRNA silencing. However, with the emerging view of 3' UTRs as platforms for regulation through RNA-binding proteins and miRNAs, we predict that more examples of cooperation - and, indeed, antagonism - between LIN41 and miRNAs will emerge, with the rules of interaction depending on the sequence or architecture of individual 3' UTRs.

\section{An enigmatic RING domain: To ubiquitylate or not to ubiquitylate?}

Ubiquitylation is a post-translational protein modification that not only results in proteasome-mediated degradation, but also alters protein function in many different ways (Komander and Rape 2012). It is catalyzed by a multisubunit enzyme complex, which obtains its substrate specificity from its E3 subunit. A large class of these E3 ligase subunits contains a characteristic RING domain, which is also found in the TRIM domain of most LIN41 proteins. Accordingly, and as an alternative to LIN41 functioning as a translational repressor, it was suggested to destabilize target proteins such as LIN-29 through polyubiquitylation (Slack et al. 2000).

However, a few observations seemed to argue against ubiquitylation as a major molecular activity of LIN41 in developmental processes. First, the putative Drosophila LIN41 dappled/wech lacks a RING domain (O'Farrell et al. 2008). Second, although screens for heterochronic mutants and let-7lf suppressors identified many LIN41lf and null alleles, no point mutations affecting the RING domain were identified (Slack et al. 2000). In contrast, several point mutations affected the NHL domain. Finally, LIN41 function in hypodermal and male tail development is highly dosage-sensitive (Del Rio-Albrechtsen et al. 2006), which would be unexpected if its major role was catalytic.

Nonetheless, ubiquitylation activity of LIN41 has now been reported in several studies. LIN41 can autoubiquitylate in vitro and in cells (Rybak et al. 2009; Loedige et al. 2012), and it has been speculated that this may be used as an autoregulatory mechanism in vivo (Del RioAlbrechtsen et al. 2006), although experimental evidence for this is currently lacking. A second validated target is Ago, although, as discussed above, the consequences of Ago ubiquitylation are unclear, with Ago destabilization not observed in most instances.

Recently, J Chen et al. (2012) identified Shc-binding protein 1 (Shcbp1) as a possible mediator of LIN41's functions in regulating mouse neural development, as discussed in an earlier section. Strikingly, Shcbp1 is ubiquity- 
lated in vivo and binds to LIN41, as determined by yeast two-hybrid and coimmunoprecipitation analysis of tagged, ectopically expressed proteins in HEK293 cells. Somewhat counterintuitively, Shcbp1 levels are reduced in embryonic neuroepithelium from LIN411f mice, and conversely, LIN41 overexpression in HEK293T cells increases the stability, and thus levels of, Shcbp1. It is, however, unclear whether stabilization and ubiquitylation of Shcbp1 are linked. This is because expression in HEK293T cells of a RING domain-less LIN41, which is inactive in promoting autoubiquitylation, continues to promote significant, albeit reduced, ubiquitylation of Shcbp1 and stabilizes Shcbp1 to almost the same degree as does wild-type LIN41. Thus, it will be important to identify the sites on Shcbp1 that are targets of ubiquitylation to test whether Shcbp1 mutated to prevent ubiquitylation is detabilized. Similarly, it will be interesting to determine the ubiquitylation status of endogenous Shcbp1, whose levels decline in sync with those of LIN41 during mouse embryonic brain development.

Taken together, it is now well documented that LIN41 has E3 ligase activity. However, it is still unclear in which contexts and to what extent ubiquitylation contributes to LIN41 function, including effects on LIN41 targets that are still elusive. Furthermore, a major question is whether the ubiquitylating activity of LIN41 represents a function separate from translational repression. Possibly, the RING and other domains of LIN41 jointly induce mRNA degradation and translational repression, as recently observed with another RING domain protein and translational repressor, MEX-3C (Cano et al. 2012).

\section{Outlook and conclusion}

Converging results from research in vertebrates and invertebrates have now helped to generate a picture of LIN41 as a key effector of the let-7 miRNA pathway that promotes cell proliferation and inhibits differentiation to control various developmental processes. The emerging fact that LIN41 is itself a translational repressor further increases the sophistication of the LIN28/let-7/LIN41 network architecture: let-7 directly represses cell cycle genes and thus proliferation (Johnson et al. 2007). To promote differentiation, it further down-regulates transcription factors that are important for progenitor states (Großans et al. 2005; Melton et al. 2010). At the same time, repression of LIN41 by let-7 derepresses cell cycle inhibitors, which provides a further break on proliferation. Finally, differentiation-inducing factors are also likely to be released from LIN41-mediated repression, although their identities need to be revealed. This dual functionrepression of direct targets and liberation of LIN41 targets from repression-might be important to achieve robust changes in gene expression and thus cell fates.

Despite the evident importance of LIN41 as an effector of let-7, we predict that LIN41 may also emerge as a critical node in developmental events not controlled by let-7. The lin-28 heterochronic gene has at least three distinct molecular activities in mammalian cells, including some not requiring let-7 (Polesskaya et al. 2007;
Rybak et al. 2008; Viswanathan et al. 2008; Cho et al. 2012), and by analogy, LIN41 might influence development by several distinct mechanisms. The widespread expression and complex biological output of LIN41, together with its intricate domain architecture, also favor such a scenario, as they suggest that the activity of LIN41 might be modulated at various levels. Nonetheless, how such modulation occurs and how LIN41 promotes translational silencing and mRNA degradation mechanistically remains to be determined. Moreover, although there is now little doubt that LIN41 can support ubiquitylation, the physiological significance of this activity requires further study. We speculate that the E3 ligase activity of LIN41-or (given the activity of overexpressed, RINGless LIN41) of a LIN41-binding partner-has more specific functions in particular processes. Examination of the rescue of LIN411f mouse phenotypes by a ubiquitylationdeficient LIN41 transgene may offer one way forward.

Finally, the Drosophila LIN41 dappled/wech is required for a functional link between integrins and the cytoskeleton-and thus muscle attachment to the body wall-by virtue of its interaction with Talin and the integrin-linked kinase ILK (Loer et al. 2008). Although it remains to be determined whether the canonical, RING finger-containing LIN41 proteins in other animals share this function, it is intriguing that endogenous TRIM71 in adult mice is also detected at sarcomeric $\mathrm{Z}$ disks of adult muscles, where it colocalizes with ILK (Loer et al. 2008).

At any rate, the recent exciting progress on the developmental, cellular, and molecular functions of LIN41 has clearly demonstrated that, beyond serving as a model miRNA target, LIN41 is a worthy subject of scientific examination in its own right.

\section{Acknowledgments}

We thank Dr. Inga Loedige and Dr. Rafal Ciosk for a critical reading of our manuscript. Work in H.G.'s laboratory is supported by the Novartis Research Foundation through the FMI Foundation, the Swiss National Science Foundation (SNF 31003A_127052 and SNF 31003A_143313), and the European Research Council (miRTurn; ERC 2419845). M.E. is SNF/SAMW Swiss MD/PhD program fellow supported by an Oncosuisse fellowship.

\section{References}

Ambros V, Horvitz HR. 1984. Heterochronic mutants of the nematode Caenorhabditis elegans. Science 226: 409-416.

Bagga S, Bracht J, Hunter S, Massirer K, Holtz J, Eachus R, Pasquinelli AE. 2005. Regulation by let-7 and lin-4 miRNAs results in target mRNA degradation. Cell 122: 553-563.

Bettinger JC, Lee K, Rougvie AE. 1996. Stage-specific accumulation of the terminal differentiation factor LIN-29 during Caenorhabditis elegans development. Development 122: 2517-2527.

Büssing I, Slack FJ, Großans H. 2008. let-7 microRNAs in development, stem cells and cancer. Trends Mol Med 14: 400409.

Büssing I, Yang J-S, Lai EC, Großhans H. 2010. The nuclear export receptor XPO-1 supports primary miRNA processing in C. elegans and Drosophila. EMBO I 29: 1830-1839.

Cano F, Bye H, Duncan LM, Buchet-Poyau K, Billaud M, Wills MR, Lehner PJ. 2012. The RNA-binding E3 ubiquitin ligase 
MEX-3C links ubiquitination with MHC-I mRNA degradation. EMBO J 31: 3596-3606.

Castello A, Fischer B, Eichelbaum K, Horos R, Beckmann BM, Strein C, Davey NE, Humphreys DT, Preiss T, Steinmetz LM, et al. 2012. Insights into RNA biology from an atlas of mammalian mRNA-binding proteins. Cell 149: 13931406.

Chang T-C, Yu D, Lee Y-S, Wentzel EA, Arking DE, West KM, Dang CV, Thomas-Tikhonenko A, Mendell JT. 2008. Widespread microRNA repression by Myc contributes to tumorigenesis. Nat Genet 40: 43-50.

Chang T, Zeitels L, Hwang H, Chivukula R, Wentzel E, Dews M, Jung J, Gao P, Dang C, Beer M, et al. 2009. Lin-28B transactivation is necessary for Myc-mediated let-7 repression and proliferation. Proc Natl Acad Sci 106: 3384-3389.

Chang H-M, Martinez NJ, Thornton JE, Hagan JP, Nguyen KD, Gregory RI. 2012. Trim71 cooperates with microRNAs to repress Cdknla expression and promote embryonic stem cell proliferation. Nat Commun 3: 923.

Chen J, Lai F, Niswander L. 2012. The ubiquitin ligase mLin41 temporally promotes neural progenitor cell maintenance through FGF signaling. Genes Dev 26: 803-815.

Chen YL, Yuan RH, Yang WC, Hsu HC, Jeng YM. 2012. The stem cell E3-ligase Lin-41 promotes liver cancer progression through inhibition of microRNA-mediated gene silencing. I Pathol 229: 486-496.

Cho J, Chang H, Kwon SC, Kim B, Kim Y, Choe J, Ha M, Kim YK, Kim VN. 2012. LIN28A is a suppressor of ER-associated translation in embryonic stem cells. Cell 151: 765-777.

Dangi-Garimella S, Yun J, Eves E, Newman M, Erkeland S, Hammond S, Minn A, Rosner M. 2009. Raf kinase inhibitory protein suppresses a metastasis signalling cascade involving LIN28 and let-7. EMBO I 28: 347-358.

Del Rio-Albrechtsen T, Kiontke K, Chiou S-Y, Fitch DHA. 2006. Novel gain-of-function alleles demonstrate a role for the heterochronic gene lin-41 in C. elegans male tail tip morphogenesis. Dev Biol 297: 74-86.

Ding X, Grosshans H. 2009. Repression of C. elegans microRNA targets at the initiation level of translation requires GW182 proteins. $E M B O J$ 28: 213-222.

Duchaine TF, Wohlschlegel JA, Kennedy S, Bei Y, Conte D Jr, Pang K, Brownell DR, Harding S, Mitani S, Ruvkun G, et al. 2006. Functional proteomics reveals the biochemical niche of C. elegans DCR-1 in multiple small-RNA-mediated pathways. Cell 124: 343-354.

Grishok A, Pasquinelli AE, Conte D, Li N, Parrish S, Ha I, Baillie DL, Fire A, Ruvkun G, Mello CC. 2001. Genes and mechanisms related to RNA interference regulate expression of the small temporal RNAs that control C. elegans developmental timing. Cell 106: 23-34.

Großans H, Johnson T, Reinert KL, Gerstein M, Slack FJ. 2005. The temporal patterning microRNA let-7 regulates several transcription factors at the larval to adult transition in $C$. elegans. Dev Cell 8: $321-330$.

Guillemot F, Zimmer C. 2011. From cradle to grave: The multiple roles of fibroblast growth factors in neural development. Neuron 71: 574-588.

Hammell CM, Lubin I, Boag PR, Blackwell TK, Ambros V. 2009. nhl-2 modulates microRNA activity in Caenorhabditis elegans. Cell 136: 926-938.

Hill DA, Ivanovich J, Priest JR, Gurnett CA, Dehner LP, Desruisseau D, Jarzembowski JA, Wikenheiser-Brokamp KA, Suarez BK, Whelan AJ, et al. 2009. DICER1 mutations in familial pleuropulmonary blastoma. Science 325: 965.

Hong Y, Roy R, Ambros V. 1998. Developmental regulation of a cyclin-dependent kinase inhibitor controls postembryonic cell cycle progression in Caenorhabditis elegans. Development 125: 3585-3597.

Johnson CD, Esquela-Kerscher A, Stefani G, Byrom M, Kelnar K, Ovcharenko D, Wilson M, Wang X, Shelton J, Shingara J, et al. 2007. The let-7 microRNA represses cell proliferation pathways in human cells. Cancer Res 67: 7713-7722.

Kanamoto T, Terada K, Yoshikawa H, Furukawa T. 2006. Cloning and regulation of the vertebrate homologue of lin41 that functions as a heterochronic gene in Caenorhabditis elegans. Dev Dyn 235: 1142-1149.

Kim J, Woo AJ, Chu J, Snow JW, Fujiwara Y, Kim CG, Cantor AB, Orkin SH. 2010. A Myc network accounts for similarities between embryonic stem and cancer cell transcription programs. Cell 143: 313-324.

Kloosterman WP, Wienholds E, Ketting RF, Plasterk RHA. 2004. Substrate requirements for let-7 function in the developing zebrafish embryo. Nucleic Acids Res 32: 6284-6291.

Komander D, Rape M. 2012. The ubiquitin code. Annu Rev Biochem 81: 203-229.

Kulkarni M, Ozgur S, Stoecklin G. 2010. On track with P-bodies. Biochem Soc Trans 38: 242-251.

Kumar MS, Lu J, Mercer KL, Golub TR, Jacks T. 2007. Impaired microRNA processing enhances cellular transformation and tumorigenesis. Nat Genet 39: 673-677.

Lagos-Quintana M, Rauhut R, Lendeckel W, Tuschl T. 2001. Identification of novel genes coding for small expressed RNAs. Science 294: 853-858.

Lagos-Quintana M, Rauhut R, Yalcin A, Meyer J, Lendeckel W, Tuschl T. 2002. Identification of tissue-specific microRNAs from mouse. Curr Biol 12: 735-739.

Lancman JJ, Caruccio NC, Harfe BD, Pasquinelli AE, Schageman JJ, Pertsemlidis A, Fallon JF. 2005. Analysis of the regulation of lin-41 during chick and mouse limb development. Dev Dyn 234: 948-960.

Lau NC, Lim LP, Weinstein EG, Bartel DP. 2001. An abundant class of tiny RNAs with probable regulatory roles in Caenorhabditis elegans. Science 294: 858-862.

Lau C-k, Bachorik JL, Dreyfuss G. 2009. Gemin5-snRNA interaction reveals an RNA binding function for WD repeat domains. Nat Struct Mol Biol 16: 486-491.

Lee RC, Ambros V. 2001. An extensive class of small RNAs in Caenorhabditis elegans. Science 294: 862-864.

Lin Y-C, Hsieh L-C, Kuo M-W, Yu J, Kuo H-H, Lo W-L, Lin R-J, Yu AL, Li W-H. 2007. Human TRIM71 and its nematode homologue are targets of let-7 microRNA and its zebrafish orthologue is essential for development. Mol Biol Evol 24: $2525-2534$.

Loedige I, Gaidatzis D, Sack R, Meister G, Filipowicz W. 2012. The mammalian TRIM-NHL protein TRIM71/LIN-41 is a repressor of mRNA function. Nucleic Acids Res 41: 518-532.

Loer B, Bauer R, Bornheim R, Grell J, Kremmer E, Kolanus W, Hoch M. 2008. The NHL-domain protein Wech is crucial for the integrin-cytoskeleton link. Nat Cell Biol 10: 422-428.

Long D, Lee R, Williams P, Chan CY, Ambros V, Ding Y. 2007. Potent effect of target structure on microRNA function. Nat Struct Mol Biol 14: 287-294.

Maller Schulman BR, Liang X, Stahlhut C, DelConte C, Stefani G, Slack FJ. 2008. The let-7 microRNA target gene, Mlin41/ Trim71 is required for mouse embryonic survival and neural tube closure. Cell Cycle 7: 3935-3942.

Melo S, Ropero S, Moutinho C, Aaltonen L, Yamamoto H, Calin GA, Rossi S, Fernandez A, Carneiro F, Oliveira C, et al. 2009. A TARBP2 mutation in human cancer impairs microRNA processing and DICER1 function. Nat Genet 41: 365-370.

Melo SA, Moutinho C, Ropero S, Calin GA, Rossi S, Spizzo R, Fernandez AF, Davalos V, Villanueva A, Montoya G, et al. 
2010. A genetic defect in Exportin-5 traps precursor microRNAs in the nucleus of cancer cells. Cancer Cell 18: 303-315.

Melton C, Judson RL, Blelloch R. 2010. Opposing microRNA families regulate self-renewal in mouse embryonic stem cells. Nature 463: 621-626.

Mondol V, Pasquinelli AE. 2012. Let's make it happen: The role of let-7 microRNA in development. Curr Top Dev Biol 99: 1-30.

Neumüller RA, Betschinger J, Fischer A, Bushati N, Poernbacher I, Mechtler K, Cohen SM, Knoblich JA. 2008. Mei-P26 regulates microRNAs and cell growth in the Drosophila ovarian stem cell lineage. Nature 454: 241-245.

Nguyen CQ, Hall DH, Yang Y, Fitch DHA. 1999. Morphogenesis of the Caenorhabditis elegans male tail tip. Dev Biol 207: 86-106.

O'Farrell F, Esfahani SS, Engström Y, Kylsten P. 2008. Regulation of the Drosophila lin-41 homologue dappled by let-7 reveals conservation of a regulatory mechanism within the LIN-41 subclade. Dev Dyn 237: 196-208.

Pasquinelli AE, Reinhart BJ, Slack FJ, Martindale MQ, Kuroda MI, Maller B, Hayward DC, Ball EE, Degnan B, Müller P, et al. 2000. Conservation of the sequence and temporal expression of let-7 heterochronic regulatory RNA. Nature 408: 86-89.

Polesskaya A, Cuvellier S, Naguibneva I, Duquet A, Moss EG, Harel-Bellan A. 2007. Lin-28 binds IGF-2 mRNA and participates in skeletal myogenesis by increasing translation efficiency. Genes Dev 21: 1125-1138.

Reinhart BJ, Slack FJ, Basson M, Pasquinelli AE, Bettinger JC, Rougvie AE, Horvitz HR, Ruvkun G. 2000. The 21-nucleotide let-7 RNA regulates developmental timing in Caenorhabditis elegans. Nature 403: 901-906.

Reinhart BJ, Weinstein EG, Rhoades MW, Bartel B, Bartel DP. 2002. MicroRNAs in plants. Genes Dev 16: 1616-1626.

Rougvie AE, Ambros V. 1995. The heterochronic gene lin-29 encodes a zinc finger protein that controls a terminal differentiation event in Caenorhabditis elegans. Development 121: 2491-2500.

Rybak A, Fuchs H, Smirnova L, Brandt C, Pohl EE, Nitsch R, Wulczyn FG. 2008. A feedback loop comprising lin-28 and let-7 controls pre-let-7 maturation during neural stem-cell commitment. Nature 10: 987-993.

Rybak A, Fuchs H, Hadian K, Smirnova L, Wulczyn E, Michel G, Nitsch R, Krappmann D, Wulczyn F. 2009. The let-7 target gene mouse lin-41 is a stem cell specific E3 ubiquitin ligase for the miRNA pathway protein Ago2. Nat Cell Biol 11: $1411-1420$.

Schulman BRM, Esquela-Kerscher A, Slack FJ. 2005. Reciprocal expression of lin-41 and the microRNAs let-7 and mir-125 during mouse embryogenesis. Dev Dyn 234: 1046-1054.

Schwamborn JC, Berezikov E, Knoblich JA. 2009. The TRIMNHL protein TRIM32 activates microRNAs and prevents self-renewal in mouse neural progenitors. Cell 136: 913-925.

Slack FJ, Ruvkun G. 1998. A novel repeat domain that is often associated with RING finger and B-box motifs. Trends Biochem Sci 23: 474-475.

Slack FJ, Basson M, Liu Z, Ambros V, Horvitz HR, Ruvkun G. 2000. The lin- 41 RBCC gene acts in the C. elegans heterochronic pathway between the let-7 regulatory RNA and the LIN-29 transcription factor. Mol Cell 5: 659-669.

Sonoda J, Wharton RP. 2001. Drosophila Brain Tumor is a translational repressor. Genes Dev 15: 762-773.

Sulston JE, Horvitz HR. 1977. Post-embryonic cell lineages of the nematode, Caenorhabditis elegans. Dev Biol 56: 110-156.

Vadla B, Kemper K, Alaimo J, Heine C, Moss EG. 2012. lin-28 controls the succession of cell fate choices via two distinct activities. PLoS Genet 8: e1002588.
Vella MC, Choi E-Y, Lin S-Y, Reinert K, Slack FJ. 2004a. The C. elegans microRNA let-7 binds to imperfect let-7 complementary sites from the lin-41 3'UTR. Genes Dev 18: 132137.

Vella MC, Reinert K, Slack FJ. 2004b. Architecture of a validated microRNA:target interaction. Chem Biol 11: 1619-1623.

Viswanathan S, Daley G, Gregory R. 2008. Selective blockade of microRNA processing by Lin-28. Science 320: 97-100.

Wulczyn FG, Cuevas E, Franzoni E, Rybak A. 2011. miRNAs need a trim: Regulation of miRNA activity by Trim-NHL proteins. Adv Exp Med Biol 700: 85-105.

$\mathrm{Xu} \mathrm{C}$, Min J. 2011. Structure and function of WD40 domain proteins. Protein Cell 2: 202-214.

Yu G, Yang Y, Tian G. 2010. Expressing and characterization of mLIN-41 in mouse early embryos and adult muscle tissues. J Mol Histol 41: 295-305.

Zhu H, Shyh-Chang N, Segrè AV, Shinoda G, Shah SP, Einhorn WS, Takeuchi A, Engreitz JM, Hagan JP, Kharas MG, et al. 2011. The Lin28/let-7 axis regulates glucose metabolism. Cell 147: 81-94. 


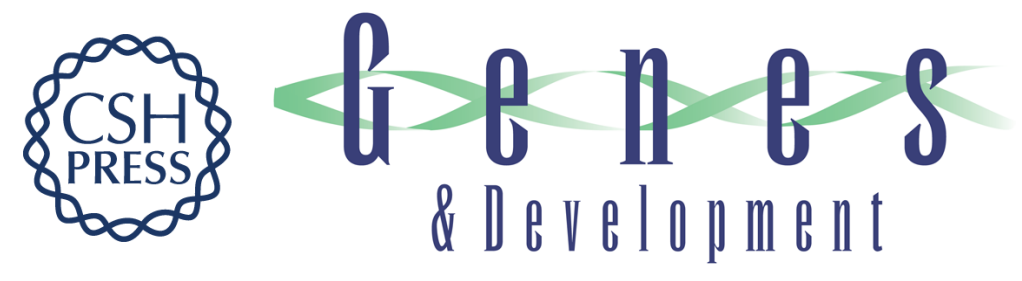

\section{LIN-41/TRIM71: emancipation of a miRNA target}

Matyas Ecsedi and Helge Großhans

Genes Dev. 2013, 27:

Access the most recent version at doi:10.1101/gad.207266.112

References This article cites 69 articles, 16 of which can be accessed free at: http://genesdev.cshlp.org/content/27/6/581.full.html\#ref-list-1

License

Email Alerting Receive free email alerts when new articles cite this article - sign up in the box at the top Service right corner of the article or click here.

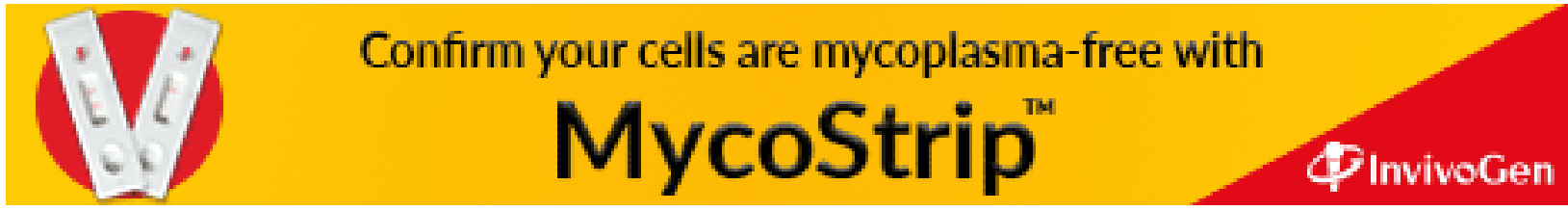

